\title{
AN UNUSUAL CASE OF PRIMARY BILATERAL RENAL LYMPHOMA: A CASE REPORT
}

\author{
Mitul Bora ${ }^{1}$, Sadhan Brata Das ${ }^{2}$, Roslin Loitongbam ${ }^{3}$, Himadri Sekhar Barthkur ${ }^{4}$, Tandra Biswas ${ }^{5}$
}

\section{HOW TO CITE THIS ARTICLE:}

Mitul Bora, Sadhan Brata Das, Roslin Loitongbam, Himadri Sekhar Barthkur, Tandra Biswas. "An Unusual Case of Primary Bilateral Renal Lymphoma: A Case Report". Journal of Evolution of Medical and Dental Sciences 2014; Vol. 3, Issue 37, August 21; Page: 9679-9684, D0I: 10.14260/jemds/2014/3251

ABSTRACT: Primary renal lymphoma (PRL) is rare. They are variants of extranodal non-Hodgkins lymphoma. Lymphomatous involvement of the kidney is often seen as a part of disseminated disease. PRL can mimic other renal lesions such as renal cell carcinoma, renal abscess, and metastasis and may have aggressive clinical course. Pathological data are scanty and prognosis is usually poor with median survival less than a year. We present a rare case of a 22 year-old male who presented with acute febrile illness, respiratory problems and acute kidney injury. The clinical diagnosis was missed partly due to absence of obvious renal symptoms like renal mass lesion, gross hematuria or abdominal pain. However, radiological investigations combined with renal histology helped in establishing the diagnosis in the present case.

KEYWORDS: Primary renal lymphoma; mild acute kidney injury; bilateral symmetrical renal enlargement.

INTRODUCTION: Lymphomas are malignant tumors characterized by the proliferation of lymphoid tissue cells. Lymphomatous involvement of the genitourinary organs occurs in $3 \%$ of the cases, most frequently in the testicles. [1] Kidneys are also an uncommon primary extranodal lymphoma (ENL) location. Primary renal lymphomas (PRL) are defined as lymphomas arising in the renal parenchyma and not invasion from an adjacent lymphomatous mass. [2]

The etiology and pathogenesis remain controversial, and there is currently no standard treatment for it. PRL usually appears in males in their sixties. PRL accounts for $0.7 \%$ of all extranodal lymphomas and represents less than $1 \%$ of all renal lesions. [3] Renal involvement is normally unilateral; an affection of both sides is unusual. Diagnosis is often delayed due to nonspecific nature of manifestations.

Flank or abdominal pain is the most frequent symptom. However, PRL can present with proteinuria or nephrotic syndrome and rapidly progress to renal failure, especially when both kidneys are affected. PRL has been associated with inflammatory and infectious chronic diseases, such as chronic pyelonephritis, Sjögren's syndrome, systemic erythematous lupus, or Epstein-Barr virus. [4]

Renal biopsy is worthwhile in patients with atypical radiological features, since it may avoid nephrectomy. We describe a patient who presented to medicine dept and then referred to nephrology units with low-grade prolonged pyrexia, progressive weight loss, mild acute kidney injury and bilateral hugely enlarged kidneys.

CASE REPORT: A 27 years old unmarried man presented to medicine emergency with 4 months h/o on $\&$ off low grade fever, weight loss $(\approx 7 \mathrm{~kg})$, abdominal pain, generalized weakness, severe anemia and productive cough. 
He also had abnormal behavior (violent, passed urine in social places), staggering gait and loss of memory 4 months back. He was treated by local doctor with some vitamins and oral methylcobalamine and referred to higher centre. He was evaluated at a tertiary care centre where he was diagnosed to be a case of acute disseminated encephalomyelitis after MRI brain and lumbar puncture. And accordingly was treated with i.v steroids.

With these treatments he gradually became symptoms free over a period of 10-12 days but his low grade fever, abdominal pain and easy fatigability persisted for which he took multiple medications from local physician including some homeopathic medications. At admission to our hospital, on examination he was conscious, oriented, febrile (temp 100.5F) with severe anemia, tachycardia, hypotension and mildly tachypnic. Urine volume was preserved.

Routine laboratory evaluation showed a blood urea nitrogen level of $31 \mathrm{mg} / \mathrm{dl}$, serum creatinine $3.1 \mathrm{mg} / \mathrm{dl}$, uric acid was $7.3 \mathrm{mg} / \mathrm{d}$ and sodium and potassium were within normal range (136meq/l and $4.1 \mathrm{meq} / \mathrm{l})$. His hemoglobin was $6.2 \mathrm{~g} / \mathrm{dl}$, total leukocyte count $13400 / \mathrm{mm} 3$, and platelet count $1.90 \times 104 / \mathrm{mm} 3$. ESR and C-reactive protein were $111 \mathrm{~mm} / \mathrm{h}$ and $211.9 \mathrm{mg} / \mathrm{dl}$. Peripheral smear revealed mild anisocytosis, mild hypochromia and no fragmented RBCs. Reticulocyte count was 3.5 percent. Serum calcium was $9.1 \mathrm{mg} / \mathrm{dl}$ and phosphorous $4.1 \mathrm{mg} / \mathrm{dl}$. Liver function tests were normal. Blood tests for malaria, typhoid and dengue were negative. Urinalysis revealed 1+ proteinuria, 2-3 $\mathrm{RBC}$ and 1-2 pus cells.

Anti-nuclear antibody and ANCA serology were negative. Chest roentgenogram showed mild right pleural effusion with patchy consolidation. Heart size was normal and there was no mediastinal lymphadenopathy. Ultrasound of abdomen revealed marked bilateral renal enlargement with homogenous cortical echogenicity. The kidneys measured $13.1 \mathrm{~cm}$ and $13.46 \mathrm{~cm}$. There was mild hepatomegaly without evidence of any focal lesion.

Hepatic echotexture, intrahepatic vascular and biliary radicles were normal. A non-contrast computed tomography (CT) scan of abdomen confirmed bilateral diffused renal enlargement with decreased attenuation suggestive of diffuse infiltrative disease. There was no evidence of hydronephrosis or intra-abdominal lymphadenopathy [Figure]. HRCT thorax revealed mild right pleural effusion with consolidation without any enlarged lymph node. Blood and urine cultures were sterile. Sputum for AFB was negative and sputum culture was also sterile. Serum LDH was very high 3615 and repeat serum uric acid and calcium were also found to be high $(10.7 \mathrm{mg} / \mathrm{dl}$ and $9.0 \mathrm{mg} / \mathrm{dl})$.

With the background of prolonged fever, severe anemia, h/o weight loss, increased uric acid, calcium, serum LHD and bilateral enlarged kidneys, suspicion for renal lymphoma or other infiltrative disease was increased and therefore renal biopsy was planned. Bone marrow aspiration and biopsy was also done which were found to be normal.

Percutaneous renal biopsy under ultrasonic guidance showed extensive infiltration of interstitium by monomorphic neoplastic cells, some of which were round with round nuclei and scanty cytoplasm. Tumor cells were arranged in a tubular pattern at places. Tumor infiltration was found confined to interstitium and tubule without glomeruli [Figure]. He was planned to put on combination chemotherapy consisting cyclophosphamide, intravenous vincristine $2 \mathrm{mg} / \mathrm{M} 2$ after consulting with a medical oncologist but was deferred due to overwhelming sepsis and multiorgan failure.

Even with all our best effort we couldn't save the patient as he developed sepsis, septic shock and eventually multiorgan failure which leads to death of the patient. 
DISCUSSION: Acute febrile illness and progressive weight loss may be because of variety of causes. Infection as a primary pathology is unlikely in our case. Bilateral symmetrical hugely enlarged kidneys may be due to bilateral hydronephrosis, cystic renal diseases, or due to malignant or benign infiltrative diseases. Rarely, it can occur in bilateral renal vein thrombosis. However, these conditions were easily ruled out on the basis of clinical features, ultrasonography, and CT scan.

CT scan showed diffuse enlargement of kidneys without any localized SOL. High serum LDH, serum calcium and uric acid with negative immunological parameters were suggestive of malignancy as the main possibility. Obstructive urinary tract pathology was ruled out with the help of ultrasound and computed tomography. His progressively increasing azotemia may be because of primary disease or may be because of sepsis and a part of multiorgan dysfunction syndrome.

As primary lymphoma is an uncommon disease hence it needs more suspicion to make a clear cut diagnosis..5,6] At least 50 cases of PRL have been reported in the literature, many of whom has been diagnosed in autopsy.[7] Kidney is not a lymphoid organ. Therefore, the status of PRL as a primary disease or the first manifestation of a rapidly progressive systemic disease is controversial Hence the very existence of lymphomas of kidney was questioned by some investigators.

The proposed pathogenetic mechanisms include: origin in the subcapsular lymphatics, seeding via hematogenous route, an extension from retro peritoneal disease or inflammatory disease with a lymphoplasmacytic infiltrate. Some pathologists tend to think PRL is a disseminated malignancy, because in 10 to $20 \%$ of cases lymphoma affects both kidneys, ${ }^{[3,6]}$ as was in the present case. Mucosa-associated lymphoid tissue renal lymphoma is probably the underlying mechanism. [8]

The role of imaging is very crucial. The most commonly encountered pattern is that of multiple soft tissue masses, ranging from 1-3 cms, with minimal enhancement after contrast compared to surrounding renal parenchyma. ${ }^{[9]}$ Therefore, a CT scan is preferred to differentiate PRL from an RCC. PRL usually appears as a hypervascularized mass with minimal and characteristic homogeneous contrast enhancement.

Contrast-enhanced computed tomography scan is the ideal test, but was avoided in this case due to progressive renal failure. MRI is currently becoming the imaging modality of choice for evaluation of renal lesions. [9] Lower signal intensity on unenhanced T1-weighted images than normal renal cortex and less enhancement on early gadolinium-enhanced images differentiates renal lymphoma from renal cell carcinoma.

Bone marrow aspiration and biopsy did not give any clue to the diagnosis. Ultimately diagnosis was made on the basis of renal histopathology. Renal biopsy clearly is the best method to establish diagnosis with high specificity and sensitivity. The sensitivity and specificity of renal biopsy are $70 \%$ to $92 \%$ and $100 \%$, respectively, with accuracy close to $90 \%$. [10]

Treatment of PRL remains controversial and mainly depends on the primary histological subtype. Role of radiation as a primary therapy in treatment of bilateral diffuse lymphoma infiltration is also controversial. It is equally unclear if it should be only treated by chemotherapy or in combination with radiation. Lack of therapeutic trials in literature has obviously compelled clinicians to adopt a practical approach and treat according to histological grade.

As it has been established that most cases of PRL are high-grade lymphoma mainly of B-cell phenotype, they are treated by CHOP or other chemotherapy regimen with or without radiotherapy. [3,7] At present, the CHOP regimen is considered the standard treatment for renal lymphoma, but only $41 \%$ of patients with advanced disease are alive and disease-free at a median follow-up of 3 years. 
Systemic chemotherapy is currently the first treatment option for PRL. Although most authors believe that the CHOP protocol should be an elective option (as it is in non-Hodgkin's BCL), there is no agreed-upon standard treatment approach for PRL.

The addition of Rituximab to the standard CHOP chemotherapy may improve the dismal outcome reported so far. However the prognosis is reported poor universally. Median survival is less than a year. [10]

CONCLUSION: The diagnosis of primary renal lymphoma needs high degree of suspicion and should be suspected if there is bilateral enlargement of the kidneys. Modern imaging techniques including MRI abdomen and renal histology have a central role in establishing the diagnosis. There is no standardized treatment for PRL. In the absence of clinical trials due to a shortage of cases, the administration of CHOP + Rituximab is probably the treatment of choice for this disease.

\section{REFERENCES:}

1. Barreto F, Dall’oglio MF, Srougi M. Renal lymphoma. Atypical presentation of a renal tumor. Int Braz J Urol. 2006 Mar-Apr; 32.

2. Hyder AO, Mahmoud RH. Primary Renal Lymphoma. Nephrology. 2007; 12: 314-5. [PubMed].

3. Freeman C, Berg JW, Cutter SJ. Occurrence and prognosis of extranodal lymphomas. Cancer 1972; 29: 252-60.

4. Pervez H, Shaikh M, Potti A, Mehdi SA. Uncommon presentations of non-Hodgkin's lymphoma: case 3-primary renal lymphoma. J Clin Oncol 2003; 21: 567-9.

5. Y. Kameoka, N. Takahashi, A. Komatsuda et al. Kidney-limited intravascular large B cell lymphoma: a distinct variant of IVLBCL? International Journal of Hematology, vol. 89, no. 4, pp. 533-537, 2009.

6. N. Sawa, Y. Ubara, H. Katori et al. Renal intravascular large B-cell lymphoma localized only within peritubular capillaries report of a case. Internal Medicine, vol. 46, no. 10, pp. 657-662, 2007.

7. Stallone G, Infante B, Manno C, Campobasso N, Pannarale G, Schena FP. Primary renal lymphoma does exist. J Nephrol 2000; 13: 367-72.

8. Jingbo Zhang, Robert AL, Ariadne Bach. Imaging of Kidney Cancer. Radiol Clin N Am.2007; 45: 119-47. [PubMed].

9. Okuno SH, Hoyer JD, Ristow K, Witzig TE. Primary renal non-Hodgkin's lymphoma: an unusual extranodal site. Cancer 1995; 75: 2258-61.

10. G Stallone, B Infante, C Manno, N Campobasso, G Pannarale, F P Schena. Primary renal lymphoma does exist: case report and review of the literature. Journal of Nephrology, vol. 13, no. 5, pp. 367-372, 2000. 


\section{CASE REPORT}

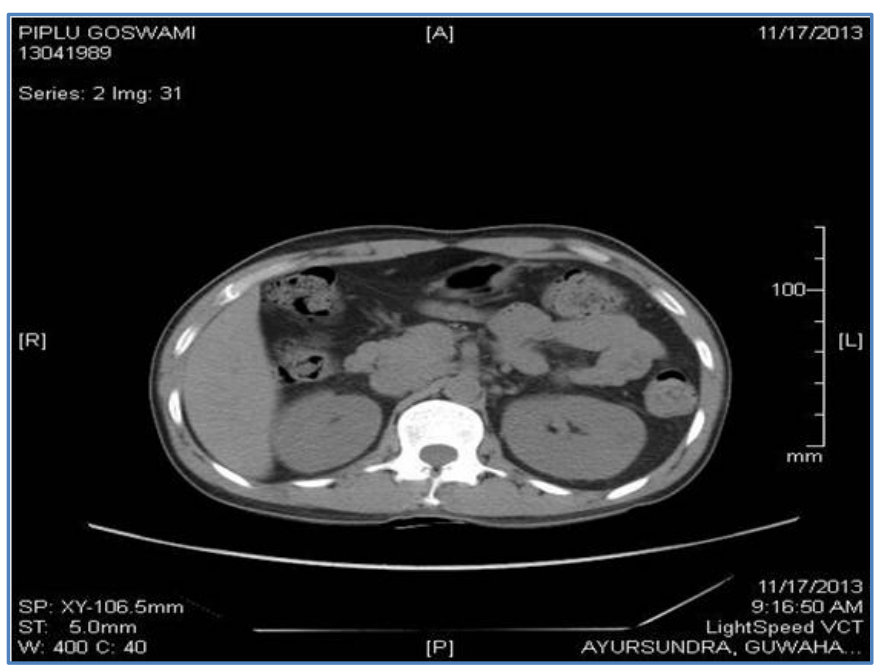

Pic 1: Showing CT scan showing bilateral hugely enlarged kidneys
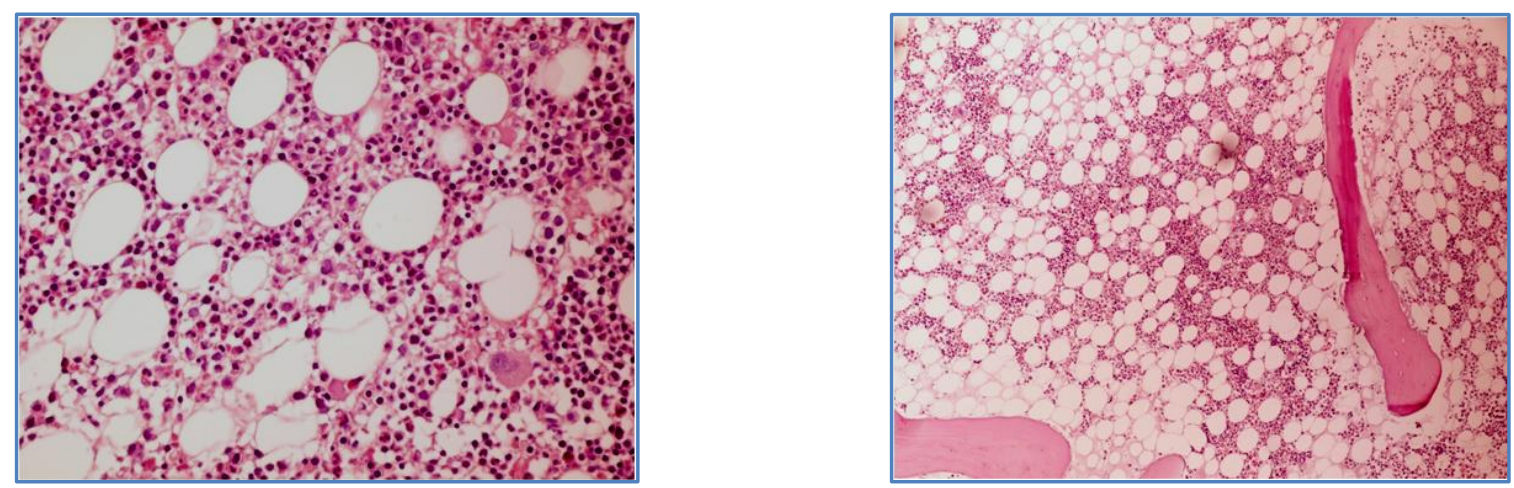

Pic 2 and 3: Bone marrow examination showing normal bone marrow
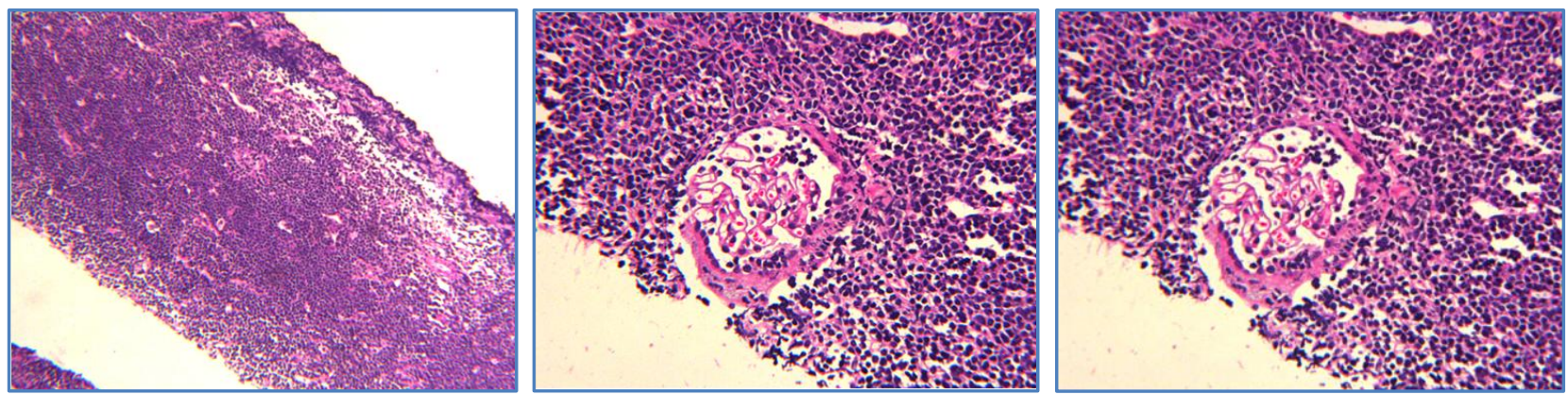

Pic 4, 5, 6: Showing renal biopsy which shows infiltration of renal tubule and interstitium by lymphomatous cells 


\section{CASE REPORT}

\section{AUTHORS:}

1. Mitul Bora

2. Sadhan Brata Das

3. Roslin Loitongbam

4. Himadri Sekhar Barthkur

5. Tandra Biswas

\section{PARTICULARS OF CONTRIBUTORS:}

1. Consultant Nephrologist, Department of Nephrology, International Hospital, Guwahati, Assam, India.

2. Consultant, Department of Medicine, International Hospital, Guwahati, Assam, India.

3. Consultant, Department of Medicine, International Hospital, Guwahati, Assam, India.

4. Consultant, Department of Medicine, International Hospital, Guwahati, Assam, India.
5. Consultant, Department of Medicine, International Hospital, Guwahati, Assam, India.

\section{NAME ADDRESS EMAIL ID OF THE CORRESPONDING AUTHOR:}

Dr. Mitul Bora,

Flat No. 504D, Uttarayan Green,

Keraikuchi, Near Hockey Stadium,

Guwahati-781040, Assam, India.

Email: boramitul@rediffmail.com

Date of Submission: 08/08/2014.

Date of Peer Review: 09/08/2014.

Date of Acceptance: 11/08/2014.

Date of Publishing: 21/08/2014. 